Revista Calidad en la Educación Superior

Programa de Autoevaluación Académica

Universidad Estatal a Distancia

ISSN 1659-4703

Costa Rica

revistacalidad@uned.ac.cr

\title{
PERCEPCIÓN DE LOS GRADUADOS DE LA ESCUELA DE CIENCIAS DE LA EDUCACIÓN DE LA UNIVERSIDAD ESTATAL A DISTANCIA (UNED) CON RESPECTO A LA FORMACIÓN RECIBIDA DURANTE LA CARRERA
}

\section{GRADUATE SCHOOL' PERCEPTION OF DISTANCE STATE UNIVERSITY (UNED) SCHOOL SCIENCES EDUCATION, REGARDING THE TRAINING RECEIVED DURING THE CAREER}

\author{
Jensy Campos Céspedes ${ }^{1}$ \\ Jennory Benavides Elizondo ${ }^{2}$
}

IV Edición

Volumen III, Número 2

Noviembre 2011

pp. $103-137$

Recibido: julio 2011

Aprobado: agosto 2011

\footnotetext{
${ }^{1}$ M.Sc-, encargada de la cátedra de Investigación de la Escuela de Ciencias de la Educación de la UNED, correo electrónico: ycampos@uned.ac.cr.

${ }^{2}$ M.Sc. Encargada de la cátedra de Informática Educativa de la Escuela de Ciencias de la Educación de la Universidad Estatal a Distancia, correo electrónico: jbenavides@uned.ac.cr
} 
Percepción de los graduados de la escuela de ciencias de la educación de la universidad estatal a distancia (UNED) con respecto a la formación recibida durante la carrera

Jensy Campos Céspedes, Jennory Benavides Elizondo

\section{Resumen}

Se presentan los resultados parciales de una investigación exploratoria desarrollada en una escuela formadora de docentes. El estudio incluyó el contacto vía telefónica con 392 graduados y el desarrollo de talleres con 93 personas graduadas de la universidad quienes llenaron un documento de consentimiento informado, participaron de un taller y llenaron una encuesta. Pese a que no se trabajó con una muestra representativa de la población graduada, sí se logró obtener información valiosa para el desarrollo de procesos de mejoramiento de la calidad educativa en los programas académicos que integran la Escuela de Ciencias de la Educación (ECE). Uno de los resultados más relevantes de este estudio identifica el interés de los graduados por contar con un espacio de formación continua por parte de la universidad. Asimismo, los graduados manifiestan altos niveles de satisfacción con la formación recibida, pese a que reconocen algunas debilidades en áreas específicas de su formación profesional inicial. De los profesionales que obtuvieron un título en la ECE la mayoría no ha continuado con estudios superiores.

Palabras claves: seguimiento a graduados, pertinencia social, formación inicial, educación superior.

\section{Summary}

These paper present partial results of an exploratory research developed into a training school for teachers. The study included telephone contact with 392 graduates and the development of workshop with 93 college graduates who completed a document of informed consent, participated in workshop and completed a survey. Although not worked with representative sample of the population graduated, they were able to obtain valuable information for developing processes to improve educational quality in academic programs that integrate the School of Education (ECE). One of the most important results of this study identifies the interest of the graduates have a space of continuous training by the university. In addition, graduates demonstrate high levels of satisfaction with the training. Professionals who obtained a degree in ECE most have not continued with higher education.

Keywords: tracking graduates, social relevance, initial training, higher education 
Percepción de los graduados de la escuela de ciencias de la educación de la universidad estatal a distancia (UNED) con respecto a la formación recibida durante la carrera

Jensy Campos Céspedes, Jennory Benavides Elizondo

Las investigaciones a propósito de la percepción de los graduados o los estudios de seguimiento a graduados son considerados elementos básicos en las unidades formadoras de profesionales que deciden hacer del mejoramiento calidad y de la pertinencia social algunos de los criterios sobre los cuales asientan su quehacer. La Escuela de Ciencias de la Educación (ECE) de la Universidad Estatal a Distancia (UNED), al igual que la mayoría de instituciones de educación superior en Costa Rica, no cuenta con sistemas de seguimiento a graduados (Berrocal, Brenes, Campos, Chaves y Seas, 2008). Por lo anterior, y como complemento a los esfuerzos que las Comisiones de Autoevaluación de las Carreras y el Programa de Autoevaluación Académica llevan a cabo, se ejecutó un estudio orientado a:

1. Identificar fortalezas y debilidades de los planes de formación de los que los graduados fueron parte.

2. Conocer la disponibilidad de los graduados para participar en actividades de desarrollo profesional e iniciativas de investigación educativa.

El interés por trabajar sostenidamente con los graduados de la ECE tiene, en primer lugar, como justificación la misión institucional, cual es brindar oportunidades de educación superior a las poblaciones que por razones socioeconómicas no tienen esa oportunidad en otras instituciones educativas.

En segundo lugar, los resultados de los proceso de autoevaluación con fines de acreditación de las carreras de la ECE, determinan la necesidad de fortalecer el 
Percepción de los graduados de la escuela de ciencias de la educación de la universidad estatal a distancia (UNED) con respecto a la formación recibida durante la carrera

Jensy Campos Céspedes, Jennory Benavides Elizondo

seguimiento a los graduados, más allá de la aplicación de la encuesta a graduandos (Berrocal, Brenes, Campos, Chávez y Seas, 2008; Campos y Madriz, 2009).

En tercer lugar, hay una necesidad impostergable de que la ECE contribuya de forma decidida en el mejoramiento de la calidad educativa mediante el fortalecimiento profesional de los docentes en ejercicio, en especial de los graduados; a la vez, el contacto permanente de la ECE con los profesionales en ejercicio, le brindaría a las carrera una mayor oportunidad de integrar las especificidades y necesidades de los profesionales en Educación de los diferentes contextos y escenarios profesionales.

\section{Resultados}

Según el informe de estudio a graduados realizado por el Centro de Investigación y Evaluación Institucional (CIEI), (2008) la Escuela de Ciencias de la Educación es la escuela de la universidad "que gradúa a más mujeres superando el $85 \%$ de sus graduados y graduadas, en este aspecto guarda ventaja en relación con las otras tres escuelas en 18 puntos porcentuales. Con respecto al estado civil un $47.7 \%$ están casadas mientras que en las otras escuelas se reportan más solteros en las graduaciones." (Sánchez, 2010, p.18). Más de 54\% del total de personas graduadas de esta escuela afirma tener personas que dependen económicamente de ellas o ellos. También tiene cuatro de las seis carreras que gradúan a más estudiantes. Sin embargo, el estudio arroja para esta Escuela, el porcentaje más 
Percepción de los graduados de la escuela de ciencias de la educación de la universidad estatal a distancia (UNED) con respecto a la formación recibida durante la carrera

Jensy Campos Céspedes, Jennory Benavides Elizondo

bajo de ocupación de sus graduados(as) con un 63,3\%, los cuales trabajan mayoritariamente en el Gobierno Central, Empresa privada e Instituciones autónomas y semi autónomas (Sánchez, 2010, p.18).

En términos globales, la ECE ha tenido una tendencia decreciente en cuanto a la cantidad de graduados, al menos en el periodo que comprende el estudio (ver tabla 1).

Tabla 1

Cantidad de graduados de las carreras de la ECE durante el periodo 2003-2008.

\begin{tabular}{lllllll}
\hline Carrera & 2003 & 2004 & 2005 & 2006 & 2007 & 2008 \\
\hline Ciencias de la Educación con énfasis Atención & & & & & & \\
Integral del Niño & 1 & 2 & & 0 & 0 & 0 \\
Administración Educativa & 56 & 40 & 57 & 36 & 41 & 51 \\
Educación Especial & 11 & 64 & 67 & 96 & 117 & 150 \\
Docencia & 170 & 141 & 211 & 168 & 193 & 179 \\
Educación Cívica & 14 & 10 & 14 & 23 & 9 & 13 \\
Educación Preescolar & 361 & 331 & 345 & 330 & 379 & 279 \\
Educación General Básica (I y II ciclos) & 797 & 595 & 769 & 757 & 662 & 603 \\
Informática Educativa & 31 & 23 & 41 & 22 & 26 & 35 \\
Total & 1441 & 1206 & 1504 & 1432 & 1427 & 1310 \\
\hline
\end{tabular}

Fuente: Centro de Investigación y Evaluación Institucional de la UNED, 2009.

Sin embargo, el comportamiento por carreras es diferente: las carreras de Administración Educativa, Educación Especial e Informática Educativa han presentado una tendencia creciente, mientras que las carreras de Educación General Básica y Educación Preescolar han decrecido. Por una parte, la carrera de Administración Educativa ha mostrado altibajos con una tendencia creciente en el último bienio. Por otra parte, la carrera de Licenciatura en Docencia muestra un comportamiento inestable durante todo el periodo estudiado. Es importante analizar la cantidad de graduados que registran cada una de las carreras de la ECE a la luz de las demandas sociales en cuanto a la producción de profesionales 
Percepción de los graduados de la escuela de ciencias de la educación de la universidad estatal a distancia (UNED) con respecto a la formación recibida durante la carrera

Jensy Campos Céspedes, Jennory Benavides Elizondo

y con respecto a los apoyos institucionales que se requieren en los procesos de formación inicial y de formación continua que las carreras deberán realizar para cumplir pertinentemente con los niveles de calidad de la formación profesional que se desea.

De los 379 graduados que se fueron contactados telefónicamente, fue posible trabajar con 93 de ellos en un taller denominado "Creación de comunidades de aprendizaje desde la investigación" que se realizó en diferentes regiones del país. Pese a que no es una muestra representativa de la población total de graduados de la ECE durante ese espacio didáctico, se aplicó una encuesta a los participantes y también se desarrollaron actividades participativas. Así, se obtuvo información de los participantes a propósito de la percepción que los graduados tenían sobre la carrera en la que fueron formados e, igualmente, se promovió la interacción entre los participantes y el intercambio de ideas acerca de las características de su ejercicio profesional y las nuevas necesidades formativas.

De la totalidad de los graduados incluidos en el estudio el $68 \%$ inició sus estudios superiores en la UNE, mientras que el $32 \%$ lo hizo en otras universidades, generalmente, en instituciones de educación superior pública, pero se han trasladaron a la UNED para finalizar su formación profesional.

En cuanto a la caracterización por sexo, se mantiene el comportamiento para la profesión docente, que es una mayoría de mujeres (78.5\%). Con respecto al grado Revista CAES Vol. II, No. 2, Año 2011 ISSN-1659-4703 
Percepción de los graduados de la escuela de ciencias de la educación de la universidad estatal a distancia (UNED) con respecto a la formación recibida durante la carrera Jensy Campos Céspedes, Jennory Benavides Elizondo

alcanzado durante su permanencia en la universidad, el de Licenciatura presenta la más alta proporción, mientras que la menor cantidad son quienes han obtenido el grado de Maestría, tal como se observa en la figura 1.

\section{Figura 1}

Distribución de los graduados incluidos en el estudio según grado académico obtenido en la ECE

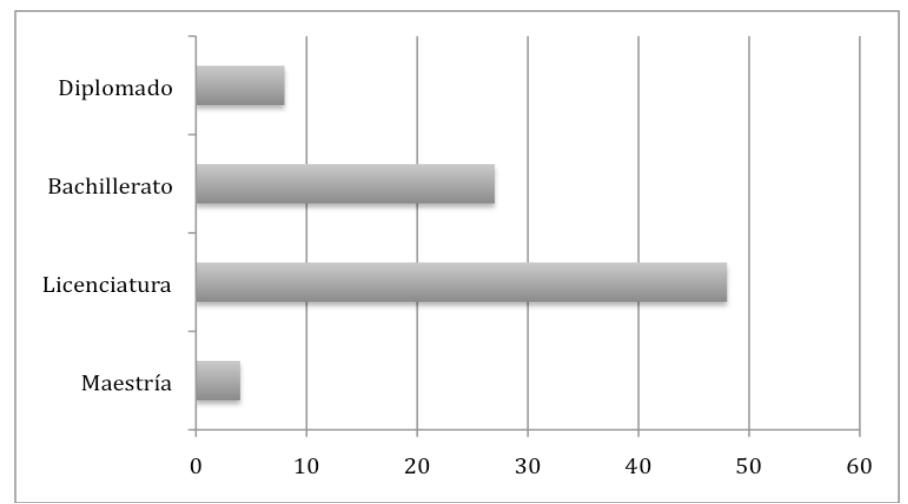

Fuente: Elaboración propia a partir de los resultados de encuesta aplicada a participantes, 2011.

En cuanto a la regularidad en la permanencia en la carrera, $43 \%$ del total ( 40 personas) interrumpió sus estudios mientras fue estudiante en la carrera. Como se aprecia en la figura 2 la mayor parte de los estudiantes interruptores deja de matricular los cursos de la carrera entre tres y cuatro cuatrimestres. Ello implica que para estas personas el tiempo de culminación de la carrera se extenderá por lo menos año y medio más de lo que se tenía previsto en el plan de estudios de la carrera (sin tomar en cuenta que la carga académica que usualmente matricula el estudiantado de la universidad está por debajo de la cantidad de créditos a la que se programa en el plan de estudios). 
Percepción de los graduados de la escuela de ciencias de la educación de la universidad estatal a distancia (UNED) con respecto a la formación recibida durante la carrera Jensy Campos Céspedes, Jennory Benavides Elizondo

Figura 2

Distribución de participantes que interrumpieron estudios según la cantidad de cuatrimestres que duro la interrupción

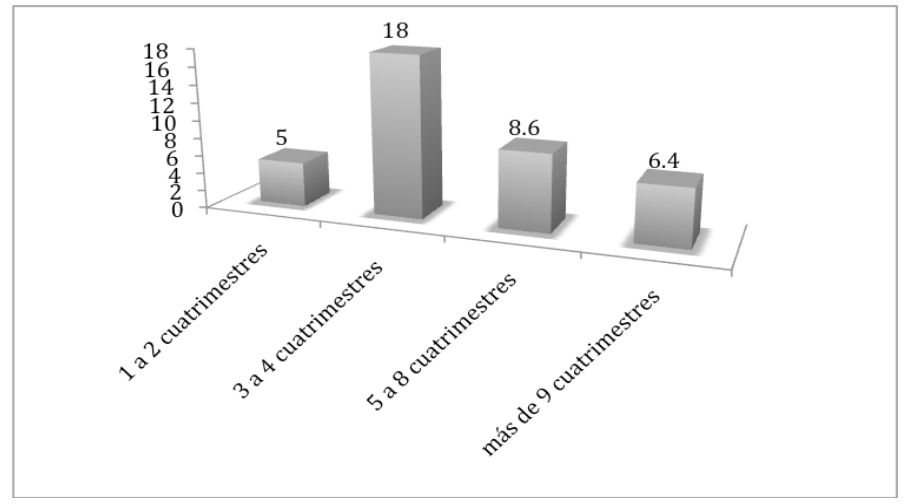

Fuente: Elaboración propia a partir de los resultados de encuesta aplicada a participantes, 2011.

Según la opinión de los graduados los motivos de la interrupción de sus estudios se deben principalmente a aspectos relacionados con la carga laboral, problemas económicos y familiares, tal como se observa en la siguiente figura.

Figura 3

Motivos argumentados por los graduados que explican la interrupción de su carrera

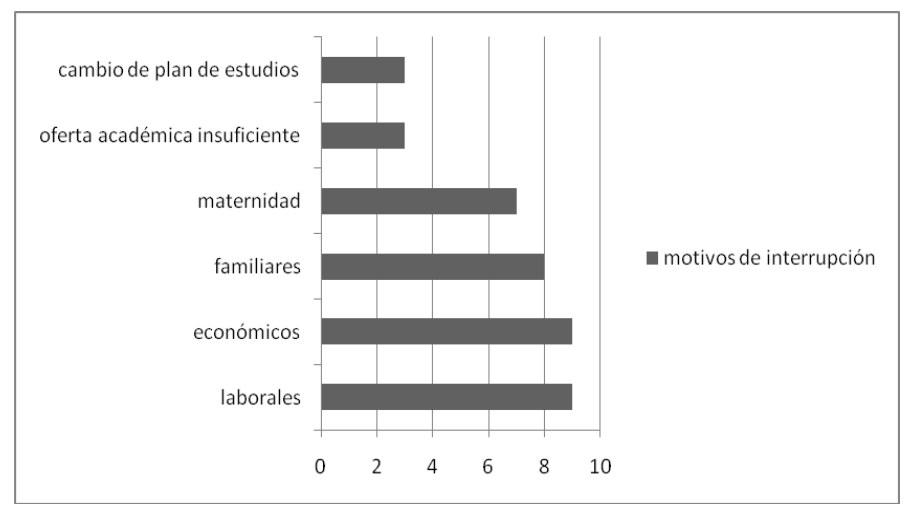

Fuente: Elaboración propia a partir de los resultados de encuesta aplicada a participantes, 2011. 
Percepción de los graduados de la escuela de ciencias de la educación de la universidad estatal a distancia (UNED) con respecto a la formación recibida durante la carrera

Jensy Campos Céspedes, Jennory Benavides Elizondo

Los motivos señalados anteriormente tienen consecuencias en el proceso de aprendizaje. En especial, la no conclusión de la carrera en el tiempo establecido en el plan de estudios, tal como ocurrió en el $45.2 \%$ de los casos incluidos en el estudio.

De los profesionales que obtuvieron un título en la UNED, la mayoría no ha continuado con estudios superiores, es decir desarrollar estudios de posgrado (55.9\% que corresponde a 52 de los entrevistados). Este es uno de los datos más relevantes para la universidad, si se proyecta ofrecer a corto o mediano plazo, opciones de actualización y profundización en temáticas relacionadas con las diversas carreras que se ofrecen en la ECE.

No obstante, fue posible obtener datos de los graduados que se mantienen como estudiantes activos en esta y otras universidades, estatales y privadas. De los 39 que continúan con estudios universitarios, 32 lo hacen en la UNED pues valoran como positivo la modalidad de estudio, el acceso a diferentes carreras, los bajos costos económicos y la aceptación de las carreras de la universidad en el espacio laboral. Las carreras que estudian los graduados que cursan actualmente otra carrera en la institución se muestran en la figura 4. 
Percepción de los graduados de la escuela de ciencias de la educación de la universidad estatal a distancia (UNED) con respecto a la formación recibida durante la carrera Jensy Campos Céspedes, Jennory Benavides Elizondo

\section{Figura 4}

Distribución de graduados que estudian otra carrera en la UNED, según carrera.

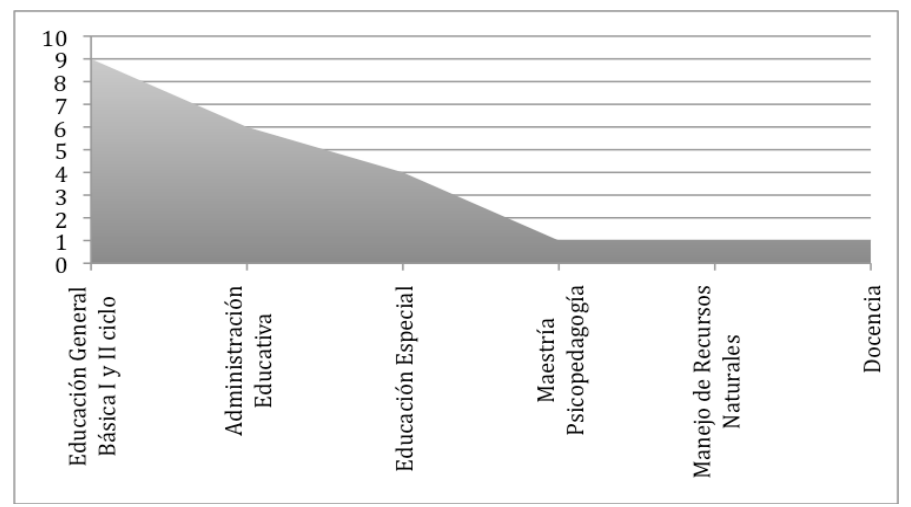

Fuente: Elaboración propia a partir de los resultados de encuesta aplicada a participantes, 2011.

Algunas de las razones por las cuales los graduados seleccionaron la carrera para estudiar, son principalmente: la modalidad a distancia; posteriormente, por una motivación personal, por el fácil acceso y por las oportunidades laborales, y en menor medida por la duración de la carrera.

Para la mayoría de los graduados, la duración de la carrera es un elemento que debe mejorarse en la ECE. Se debe tratar de disminuir los tiempos de culminación. En este sentido, los graduados indicaron que entre los factores que los afectaron negativamente para cumplir con el tiempo estipulado para finalizar el plan de estudios se encuentran en primer lugar la resistencia por matricular algunos cursos, la imposibilidad de matricular bloques concretos, así como la 
Percepción de los graduados de la escuela de ciencias de la educación de la universidad estatal a distancia (UNED) con respecto a la formación recibida durante la carrera

Jensy Campos Céspedes, Jennory Benavides Elizondo

presencia de problemas personales (compromisos familiares y maternidad) tal como se expresa en la figura 5.

\section{Figura 5}

Aspectos que afectaron negativamente el cumplimiento del tiempo de culminación establecido en el plan de estudios.

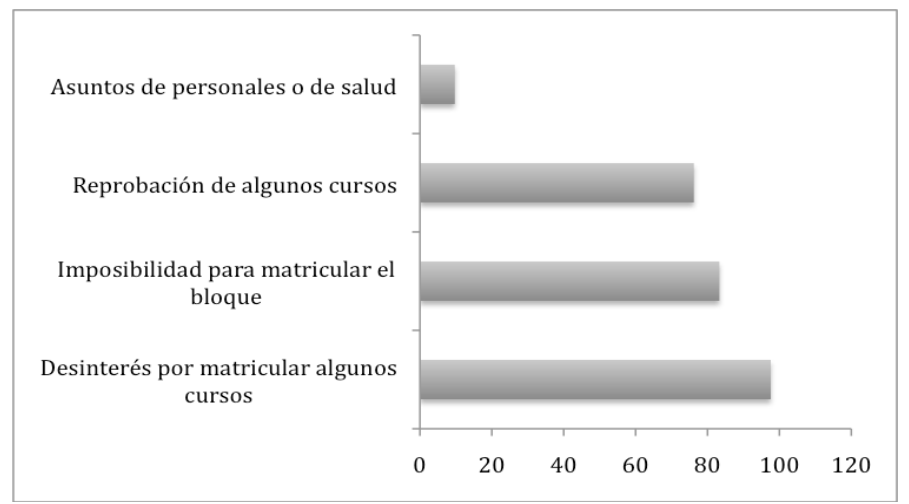

Fuente: Elaboración propia a partir de los resultados de encuesta aplicada a participantes, 2011.

Para $74 \%$ de los encuestados, la carrera que matricularon era la que deseaba estudiar y les gustó, mientras que $2 \%$ si deseaba estudiar su carrera pero no le gustó. Un $21 \%$ indicó que no deseaba estudiar esa carrera, pero que al final le gustó; finalmente, $1 \%$ dijo que no quería estudiarla y nunca le gustó aunque sí se graduó.

En el estudio realizado se les solicitó a los graduados que le asignaran una calificación a cada uno de los elementos claves del proceso formativo que se les brindó en la carrera cursada en la ECE. Para dicha calificación se utilizó una 
Percepción de los graduados de la escuela de ciencias de la educación de la universidad estatal a distancia (UNED) con respecto a la formación recibida durante la carrera

Jensy Campos Céspedes, Jennory Benavides Elizondo

escala de 1 a 5 en donde el cinco era la valoración más alta posible y el 1 la más

baja (ver tabla 2).

Tabla 2

Calificación asignada por los graduados a cada uno de los elementos clave durante su experiencia académica en la ECE (escala de 1 a 5).

\begin{tabular}{|c|c|c|c|c|c|}
\hline Elemento & Valid & Missing & Promedio & Mediana & Moda \\
\hline Bibliografía utilizada & 92 & 1 & 4.4 & 5 & 5 \\
\hline Plan de estudios & 92 & 1 & 4.3 & 4 & 5 \\
\hline $\begin{array}{l}\text { Asesoría y atención por parte del personal } \\
\text { administrativo }\end{array}$ & 89 & 4 & 4.3 & 4 & 5 \\
\hline Entrega de los materiales didácticos & 91 & 2 & 4.3 & 5 & 5 \\
\hline Información suministrada & 92 & 1 & 4.2 & 4 & 4 \\
\hline $\begin{array}{l}\text { Calidad de los materiales impresos y } \\
\text { audiovisuales }\end{array}$ & 92 & 1 & 4.2 & 4.5 & 5 \\
\hline Calidad de los cursos teóricos & 90 & 3 & 4.2 & 4 & 5 \\
\hline Calidad de los cursos prácticos & 91 & 2 & 4.1 & 4 & 5 \\
\hline Duración de la carrera & 90 & 3 & 4.0 & 4 & 4 \\
\hline $\begin{array}{l}\text { Asesoría y atención por parte del personal } \\
\text { docente }\end{array}$ & 91 & 2 & 3.9 & 4 & 4 \\
\hline Tutorías presenciales & 92 & 1 & 3.8 & 4 & 5 \\
\hline Calidad de los materiales digitales & 85 & 8 & 3.8 & 4 & 5 \\
\hline Importancia que se le da a la investigación & 92 & 1 & 3.6 & 4 & 5 \\
\hline $\begin{array}{l}\text { Calidad de los cursos con componentes } \\
\text { virtuales }\end{array}$ & 81 & 12 & 3.6 & 4 & 4 \\
\hline Tutorías virtuales & 78 & 15 & 3.5 & 4 & 4 \\
\hline
\end{tabular}

Fuente: Elaboración propia a partir de encuesta realizada a graduados, 2009.

Con respecto a la calificación que los graduados brindaron a algunos de los servicios clave de la universidad, se notó que lo que más valoran es la bibliografía utilizada y la entrega de los materiales. En este sentido, indican que les gustó mucho la mayoría de los textos porque lograban comprender fácilmente los contenidos de las asignaturas. 
Percepción de los graduados de la escuela de ciencias de la educación de la universidad estatal a distancia (UNED) con respecto a la formación recibida durante la carrera

Jensy Campos Céspedes, Jennory Benavides Elizondo

En segundo lugar, los graduados ubican la calidad de las tutorías presenciales; según ellos, en algunos cursos, las tutorías presenciales no satisficieron sus expectativas por lo que ellos optaban por no asistir y centrarse en el estudio de los materiales suministrados por la universidad. Los servicios con baja calificación fueron las tutorías virtuales y la calidad de los cursos con componentes virtuales. En los talleres algunos de los participantes manifestaron no haberse sentido cómodos en los cursos virtuales debido a que no siempre todos los estudiantes contaban con acceso a internet en su domicilio. De allí la importancia que la institución redoble esfuerzos para facilitar al estudiantado alternativas que le faciliten el acceso a internet ya sea mediante convenios interinstitucionales o mediante el fortalecimiento de centros de cómputo en los centros universitarios.

En cuanto a la calificación que los graduados asignaron a otros temas relacionados con su proceso formativo, como la equidad de género en la institución, se desprende que la mayoría califica muy alto a la institución en este sentido, al igual que el respeto por la religión, etnia y orientación sexual. Entre tanto, califican más bajo lo referido al uso de las tecnologías de la información y la comunicación (TIC), la investigación y la innovación. Esta información es congruente con algunos de los vacíos en la formación que los graduados identifican tras haberse graduados de alguna de las carreras de la ECE.

Según los graduados participantes en el estudio, es necesario que la UNED y las carreras de Educación fortalezcan y enriquezcan los planes de estudio en dichas variables ya que ellos sienten que la formación que recibieron en esos aspectos es Revista CAES Vol. II, No. 2, Año 2011 ISSN-1659-4703 
Percepción de los graduados de la escuela de ciencias de la educación de la universidad estatal a distancia (UNED) con respecto a la formación recibida durante la carrera

Jensy Campos Céspedes, Jennory Benavides Elizondo

deficitaria de cara a las condiciones que experimentan en los contextos educativos.

Un indicador importante de reconocer es el grado de satisfacción que tienen los graduados respecto de esta universidad. Con base en las cifras recabadas, se infiere que la mayoría de los graduados quedan complacidos con la oferta académica y los diferentes servicios que ofrece esta casa universitaria, tal como se observa en la figura 6 .

Figura 6

Distribución de participantes según grado de satisfacción con la carrera estudiada

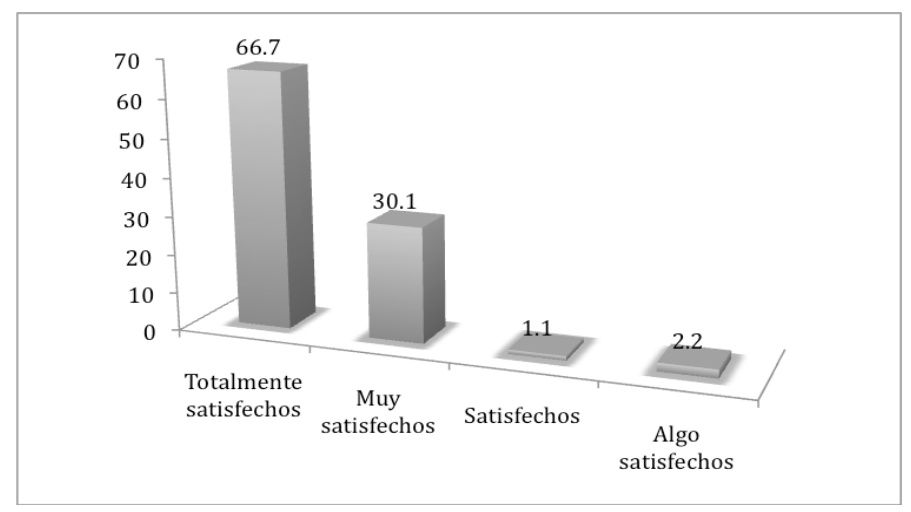

Fuente: Elaboración propia a partir de encuesta realizada a graduados, 2009.

Hasta el momento, las interrogantes y las respuestas han sido referidas a elementos que tienen relación con su permanencia como estudiantes de la universidad. No obstante, es de igual pertinencia conocer si tales graduados, después de recibir su título universitario, lograron colocarse laboralmente en el campo que obtuvo la especialidad. 
Percepción de los graduados de la escuela de ciencias de la educación de la universidad estatal a distancia (UNED) con respecto a la formación recibida durante la carrera

Jensy Campos Céspedes, Jennory Benavides Elizondo

En esa línea, es satisfactorio conocer que el $76.6 \%$ de los graduados incluidos en el estudio responde que sí trabaja en el campo laboral, cuya base es la carrera en que se graduaron. Sin embargo el $23.7 \%$ de los participantes indican lo contrario.

Con respecto a la jornada de tiempo que laboran los graduados incluidos en la investigación, se encontró que la mayoría labora tiempo completo aunque un porcentaje importante labora más de la jornada de tiempo completo en actividades asociadas a la formación que recibieron en la UNED, tal como se muestra en la siguiente figura.

\section{Figura 7}

Distribución de los participantes según jornada laboral que desempeñan

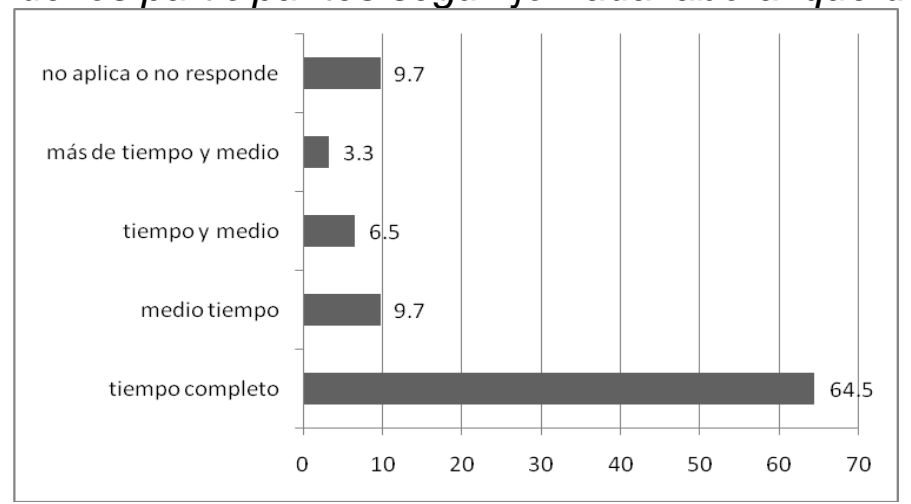

Fuente: Elaboración propia a partir de los resultados de la encuesta aplicada a los participantes.

La anterior información corrobora que ya mayoría de los estudiantes de la ECE tiene un trabajo con una jornada laboral a tiempo completo, lo que es importante considerar en todas las propuestas pedagógicas y modalidades educativas de las carreras que se ofrecen, al igual que en los diferentes servicios de apoyo.

Con respecto a la condición laboral de los graduados incluidos en el estudio se encontró que la mayoría de ellos se encuentran laborando en condición de 
Percepción de los graduados de la escuela de ciencias de la educación de la universidad estatal a distancia (UNED) con respecto a la formación recibida durante la carrera

Jensy Campos Céspedes, Jennory Benavides Elizondo

propietarios de una plaza en el Ministerio de Educación Pública (observar figura 8). Es importante tomar en cuenta que los graduados incluidos en el estudio obtuvieron su título durante el quinquenio 2004- 2009 y algunos de ellos ingresaron a la carrera cuando ya se encontraban laborando para el MEP.

Figura 8

Porcentaje de graduados incluidos en el estudio según condición laboral

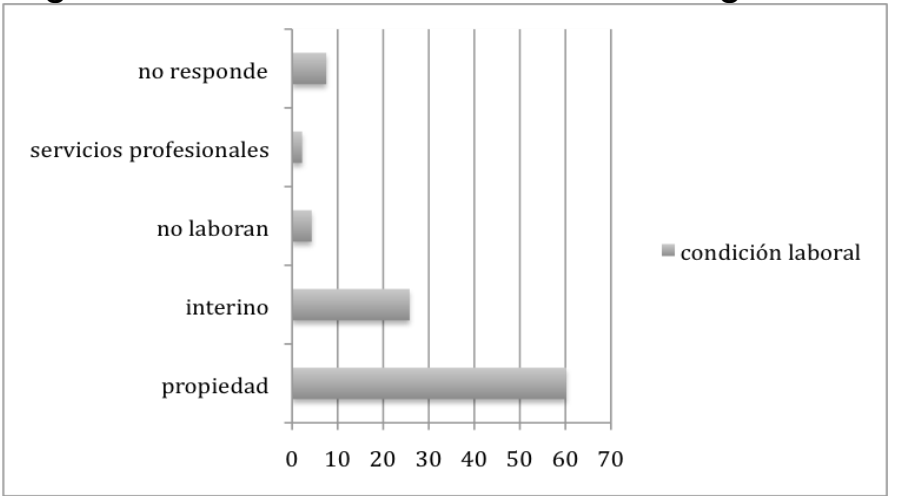

Fuente: Elaboración propia a partir de los resultados de la encuesta aplicada a los participantes.

Con respecto al grado de relación entre la carrera que curso con su trabajo actual, como se observa en la figura 9, la mayoría de los participantes indican que existe una total relación entre la formación recibida y sus desempeño laboral. Ello resulta importante, ya que gran parte de las personas graduadas se desempeñan como docentes, sin embargo, algunos no están laborando o se desempeñan en ocupaciones diferentes. 
Percepción de los graduados de la escuela de ciencias de la educación de la universidad estatal a distancia (UNED) con respecto a la formación recibida durante la carrera

Jensy Campos Céspedes, Jennory Benavides Elizondo

Figura 9

Grado de relación entre la formación recibida y el desempeño laboral actual, según la percepción de los participantes.

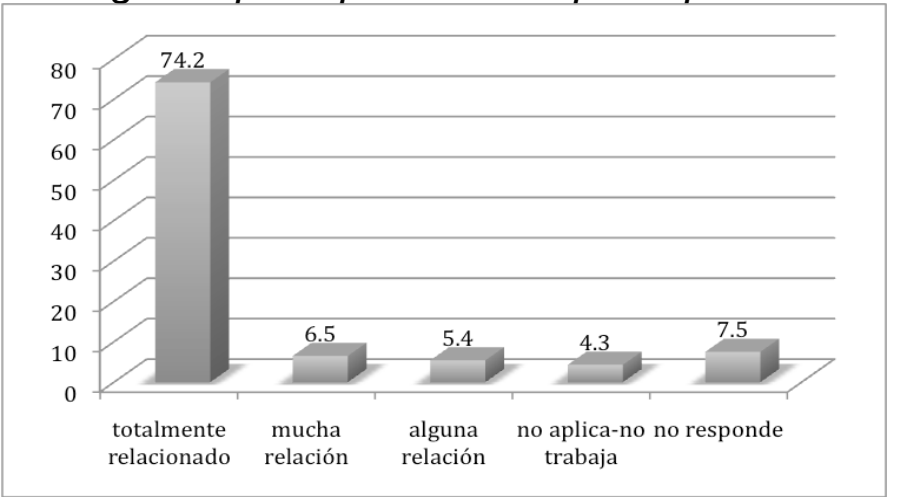

Fuente: Elaboración propia a partir de los resultados de la encuesta aplicada a los participantes.

Con respecto a la percepción de los graduados sobre si la carrera cursada dejó algunos vacíos en su formación para el $63.4 \%$ de los graduados incluidos en el estudio eso la respuesta es afirmativa, mientras que para el $35.5 \%$ la respuesta es negativa.

De los que respondieron afirmativamente, se obtuvieron los más altos porcentajes para los siguientes factores: práctica en la parte docente; tecnología educativa, investigación así como estrategias de enseñanza aprendizaje. 
Percepción de los graduados de la escuela de ciencias de la educación de la universidad estatal a distancia (UNED) con respecto a la formación recibida durante la carrera Jensy Campos Céspedes, Jennory Benavides Elizondo

Figura 10

Áreas temáticas que requieren fortalecimiento según los graduados incluidos en el estudio.

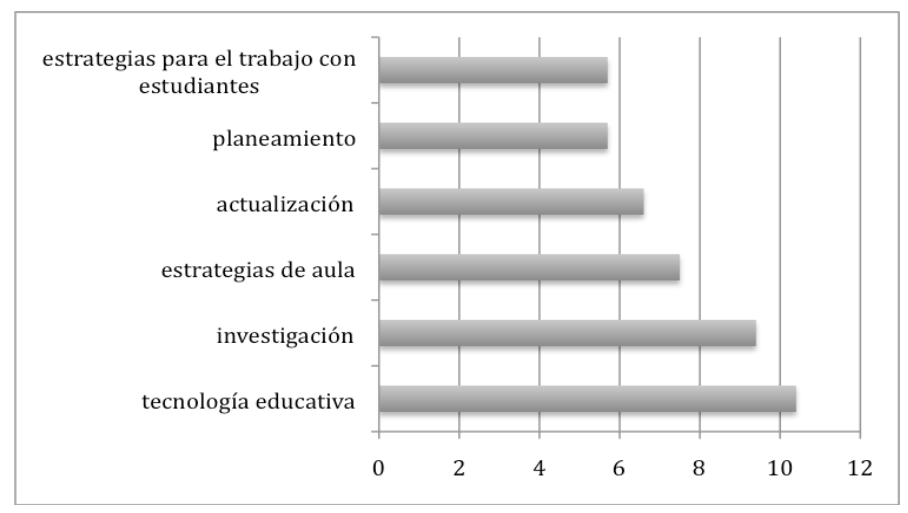

Fuente: elaboración propia a partir de los resultados de la encuesta, 2011.

Los anteriores elementos, considerados por los graduados como áreas débiles en su formación, están muy relacionados con los aspectos que ellos indicaron como necesario de ser reforzado dentro de la carrera que estudiaron en la UNED, tal como se expresa en la figura 11.

Figura 11

Opiniones de los participantes con respecto a los aspectos de los planes formativos que deben ser mejorados en la UNED

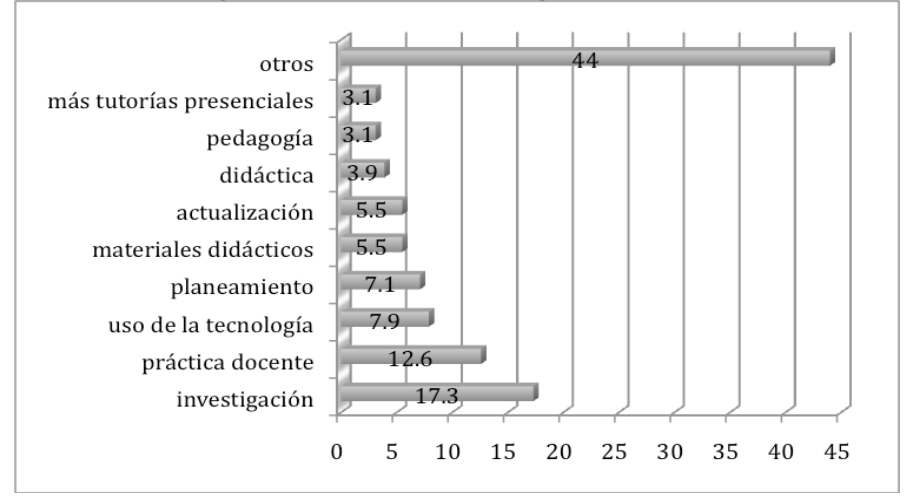

Fuente: elaboración propia a partir de los resultados de la encuesta, 2011. 
Percepción de los graduados de la escuela de ciencias de la educación de la universidad estatal a distancia (UNED) con respecto a la formación recibida durante la carrera Jensy Campos Céspedes, Jennory Benavides Elizondo

Según los graduados entre los aspectos que deben mejorarse en la formación que recibieron en la ECE se tiene la formación para la investigación. Este es seguido por la práctica docente en el aula, el uso de la tecnología, la formación para hacer planeamiento. Tal como se muestra en la figura 12.

Figura 12

Aspectos necesarios de ser mejorados por las carreras de la ECE, según opinión de los participantes

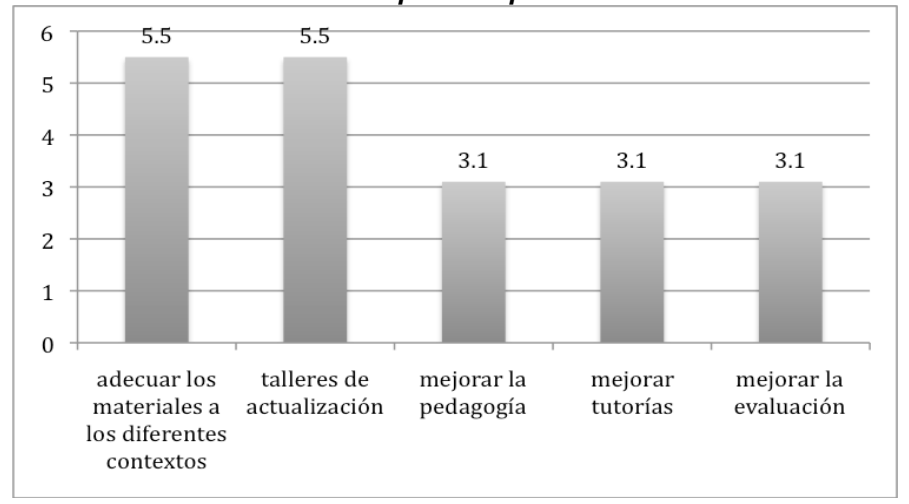

Fuente: Elaboración propia a partir de encuesta a participantes, 2011.

La universidad realiza importantes esfuerzos para adecuar materiales a las necesidades de los estudiantes en las diferentes modalidades que ofrece (a distancia, bimodal (distancia y en línea) y el e-learning (totalmente en línea)); no obstante, existen debilidades en muchos ámbitos que son mencionadas por los graduados. Sobresale el hecho de que los graduados identifiquen como uno de los aspectos a mejorar el uso de las tecnologías para el desarrollo de competencias tecnológicas por parte del estudiantado, en este sentido es vital que la institución maximice esfuerzos para evaluar el uso que se le está dando a los recursos tecnológicos en función de los procesos de aprendizaje de los estudiantes. 
Percepción de los graduados de la escuela de ciencias de la educación de la universidad estatal a distancia (UNED) con respecto a la formación recibida durante la carrera Jensy Campos Céspedes, Jennory Benavides Elizondo

En este sentido, otros trabajos desarrollados en la UNED han llamado la atención sobre la necesidad de fortalecer las competencias tecnológicas de los docentes y sobre desarrollar un modelo de calidad para la educación en línea en el que se involucren no solo el diseño y desarrollo de los cursos con algún componente de virtualidad sino también la gestión tecnológica (Campos y Solano, 2010; Brenes, Campos y Solano, 2010).

Otro de los aspectos señalado como necesarios de fortalecer dentro de los procesos formativos de las carreras de la ECE se encuentra la formación práctica. Es importante indicar que la práctica profesional en las carreras se realiza, generalmente para algunas de las carreras, como una práctica terminal en la que se espera que el estudiantado al finalizar el plan de estudios desarrolle una práctica en una institución educativa, por al menos, un semestre y con ello logre aplicar los conocimiento y dominios desarrollados durante los cursos que integran el plan de estudios. No obstante, tanto la literatura como los graduados indican la importancia de que a lo largo del proceso formativo exista una riqueza de estrategias didácticas que le permitan al estudiante acercarse al espacio profesional y al quehacer profesional. De allí que resulte valioso para la ECE el desarrollo de estrategias, como escuela, para la consecución de un banco de centros de práctica y que el componente práctico se constituya en un eje curricular en todos los programas académicos que se ofrece. La práctica como acción pedagógica concurrente permitiría a los estudiantes hacer un proceso de inserción Revista CAES Vol. II, No. 2, Año 2011 ISSN-1659-4703 
Percepción de los graduados de la escuela de ciencias de la educación de la universidad estatal a distancia (UNED) con respecto a la formación recibida durante la carrera

Jensy Campos Céspedes, Jennory Benavides Elizondo

progresivo al ejercicio profesional y conocer las realidades específicas de diferentes escenarios profesionales.

Cabe destacar que los graduados no solo mencionan el continuar actualizándose en su área laboral, sino que buscan hacerlo en otros planos. De igual manera, requieren oportunidades para mejorar sus prácticas laborales. Así lo evidenció la cantidad de graduados que dijeron estar interesados en programas de formación continua (87, lo que corresponde a $93.5 \%$ del total). Solo tres personas dijeron no estar interesadas en tal formación. Tal como lo muestra la figura 13 los graduados manifiestan desear formación en aspectos relacionados con el uso de las tecnologías de la Información y la comunicación (TICs), también en lo referido a la detección y abordaje de problemas de aprendizaje asimismo los elementos referidos con el planeamiento didáctico y la psicopedagogía.

Figura 13

Áreas en las que los graduados indican desear formación continua

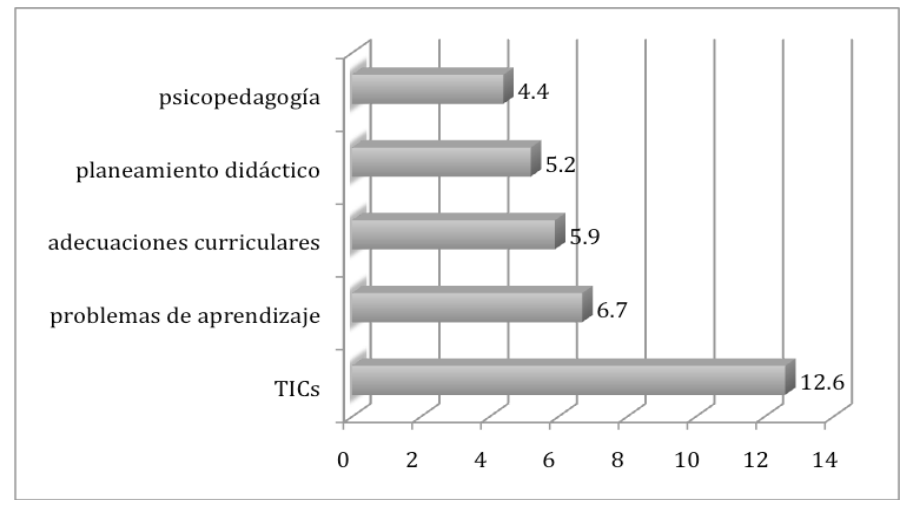

Fuente: Elaboración propia a partir de los resultados de la encuesta a los participantes, 2011. 
Percepción de los graduados de la escuela de ciencias de la educación de la universidad estatal a distancia (UNED) con respecto a la formación recibida durante la carrera

Jensy Campos Céspedes, Jennory Benavides Elizondo

Necesidades educativas, técnicas de enseñanza y temas legales fueron mencionadas, cada uno de ellos, por cinco entrevistados. Con porcentajes menores se nombraron: manejo de límites, actualizaciones, nuevas metodologías, evaluación, desarrollo social, idiomas, aprendizaje por competencias, alternativas a la diversidad, matemáticas, formación ciudadana, ecología, medio ambiente, lenguaje señas, nuevos paradigmas de la educación, coordinación, entre otros.

Al consultarles si les interesaría formar parte de una red de personas graduadas de la UNED, 90 individuos (96.8\%) respondieron afirmativamente. Solo dos señalaron no estar interesados y uno no respondió.

Se consideran como beneficios el ser parte de una red de graduados (porcentajes obtenidos con base en 178 respuestas ya que la pregunta permitía respuesta múltiple) porque podría existir mayores oportunidades de actualización profesional y desarrollar mayores conocimientos. En la figura 14 se detallan las razones expuestas por los participantes por las cuales consideran importante participar en una red de graduados de la ECE. 
Percepción de los graduados de la escuela de ciencias de la educación de la universidad estatal a distancia (UNED) con respecto a la formación recibida durante la carrera Jensy Campos Céspedes, Jennory Benavides Elizondo

\section{Figura 14}

Razones esgrimidas por los participantes sobre la importancia de formar parte de una red de graduados de la ECE

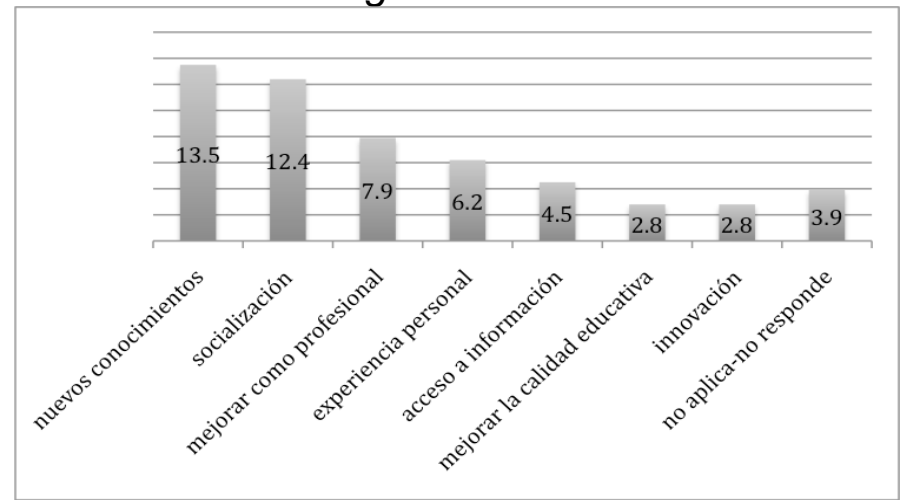

Fuente: Elaboración propia a partir de encuesta a participantes, 2011.

Para ampliar el tema del mejoramiento, algunos de los comentarios de los graduados que respondieron a la entrevista indicaban que se requerían más investigaciones en el campo educativo (cuatro personas). Además se cita que deben ser más participativas (tres personas. Algunos participantes manifiestan que: "la web puede ser útil para crear centros de opinión"; "la investigación debe ser aplicada a la realidad socioeconómica del país"; "debe darse un acompañamiento al educador; además de una mayor coordinación con el MEP;" "se necesita más apoyo a los graduados"; "es necesario que la universidad realice un análisis y reforma en el material de evaluación"; "se requiere del fortalecimiento de talleres para la actualización docente". "Es importante una mayor incorporación y aprovechamiento de las TICs".

Otras opiniones de los entrevistas apuntan a componentes como: la realización de reuniones con docentes de las diferentes áreas, que las sesiones sean entre semana y no solo los fines de semana, darles capacitación a docentes que tienen 
Percepción de los graduados de la escuela de ciencias de la educación de la universidad estatal a distancia (UNED) con respecto a la formación recibida durante la carrera

Jensy Campos Céspedes, Jennory Benavides Elizondo

muchos años de experiencias para que se actualicen, la formación del docente en material legal, el visualizar la educación como un continuo proceso de aprendizaje, que haya una selección previa de los participantes, que demuestren compromiso con sus procesos de aprendizaje para que lleguen a merecerse el título.

También se considera que haya un enfoque diferente al abordaje de la investigación, generar nuevas ofertas educativas, el tema de las matemáticas en acción, la creación de una oferta formativa para seguir capacitándose, que exista una buena comunicación y accesibilidad a la información, mejorar el diálogo para erradicar fallas en las tutorías, mejorar los procesos de práctica docente, que los temas sean diversos y así se de la posibilidad de formar subgrupos y que las propuestas sean más activas y menos memorísticas.

Al consultar a los graduados acerca del tema de la investigación y si ha desarrollado actividades en esta línea en su desempeño docente, $45.2 \%$ dice que sí las realizan, mientras que $53.8 \%$ no lo hacen. Ante la consulta que se les efectúo a los graduados referente a que si dentro de sus funciones se contemplaban acciones de investigación $47.3 \%$ de ellos contestaron en forma afirmativa y $46.2 \%$ señaló que no era parte de sus obligaciones laborales.

En lo que refiere a dedicar tiempo para realizar investigación en su quehacer profesional, tal como se observa en la figura 15 , son muy pocos los graduados que dedican tiempo de su jornada laboral para realizar actividades investigativas. 
Percepción de los graduados de la escuela de ciencias de la educación de la universidad estatal a distancia (UNED) con respecto a la formación recibida durante la carrera Jensy Campos Céspedes, Jennory Benavides Elizondo

Figura 15

Distribución de los participantes, según disponibilidad de tiempo laboral para investigar

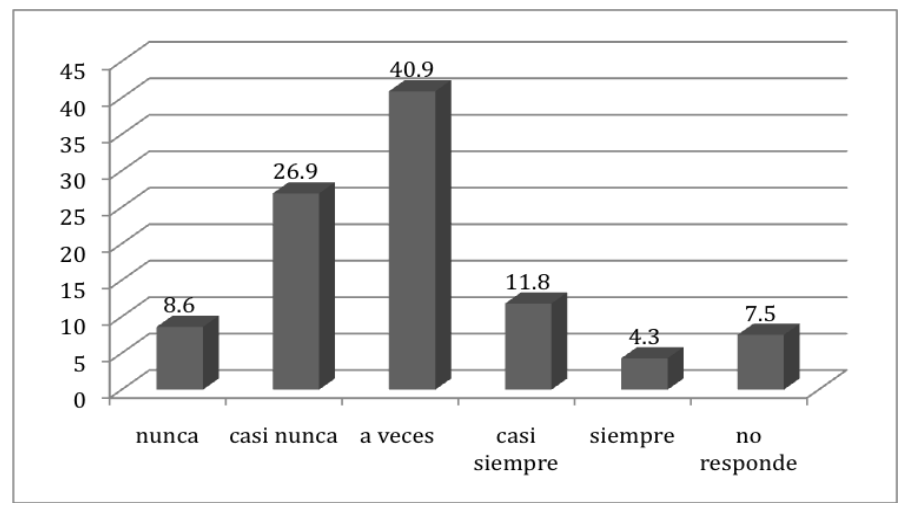

Fuente: Elaboración propia a partir de los resultados de la encuesta aplicada, 2011.

Aun cuando un porcentaje significativo de los profesionales entrevistados (45.2\%) dicen realizar actividades de investigación, no hay concordancia con la información que se compila al preguntar el tiempo dedicado a la misma, donde el $40.9 \%$ indica que a veces lo dedica. Queda la interrogante en qué aspectos específicos de sus áreas de trabajo, ponen en práctica los procesos de investigación; asimismo, resulta interesante profundizar investigando acerca de los usos que le dan los graduados a las investigaciones que realizan y específicamente para qué le sirven los resultados de estos procesos en términos del mejoramiento de la calidad educativa.

Sobre el tema de la investigación que los graduados realizan, en los talleres ejecutados con esta población se analizó que el tiempo destinado a la investigación lo hacen aquellos docentes que en este momento están cursando estudios de educación superior ya sea de grado o posgrado, por lo que la investigación que se realiza obedece a actividades académicas incluidos dentro de Revista CAES Vol. II, No. 2, Año 2011 ISSN-1659-4703 
Percepción de los graduados de la escuela de ciencias de la educación de la universidad estatal a distancia (UNED) con respecto a la formación recibida durante la carrera

Jensy Campos Céspedes, Jennory Benavides Elizondo

sus proceso formativos, más que el cumplimiento de una función profesional del docente.

Además, hay que valorar a qué tipo de investigaciones hacen referencia los profesionales que se entrevistan, ya que la mayoría dice no tener experiencia como integrantes en procesos de investigación (62.7\% señalan que no, el 31.2\% que sí y dos personas no responden). En este sentido, se exploró en los talleres con esta población y se logró extraer información que apunta hacia una concepción de investigación como la realización de revisiones de literatura y búsqueda de materiales o contenidos que permitan el desarrollo de los temas de estudio con el grupo de estudiantes a su cargo.

Es evidente que los graduados entrevistados no tienen experiencias en publicaciones, $89.2 \%$ personas nunca ha elaborado una publicación.

Continuando con el análisis, los procesos de investigación son valorados por los entrevistados, como uno de los aspectos que deben ser considerados en ofertas de capacitación ya que lo definen como una debilidad, en su formación universitaria, en la carrera que concluyeron. Al consultarles si tienen disponibilidad para recibir capacitación en investigación, el mayor porcentaje de los noventa y tres que responden a este ítem, indican que sí la tienen (ochenta y dos personas, 88.2\%). Solo siete, dicen no contar con esta disponibilidad y cuatro no responden (ver figura 16). 
Percepción de los graduados de la escuela de ciencias de la educación de la universidad estatal a distancia (UNED) con respecto a la formación recibida durante la carrera Jensy Campos Céspedes, Jennory Benavides Elizondo

Figura 16

Distribución de los participantes según disponibilidad a recibir formación continua en el campo de la investigación

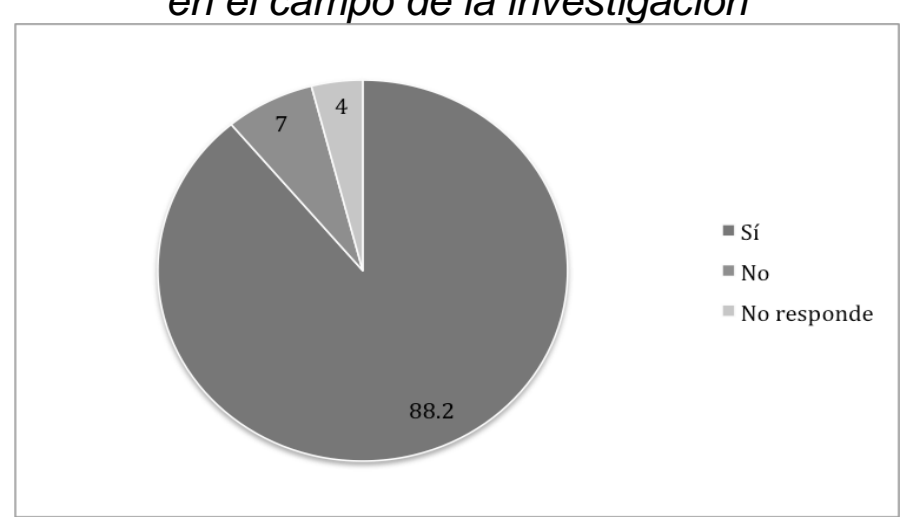

Fuente: Elaboración propia con base en resultados de encuesta a participantes, 2011.

\section{Figura 17}

Razones por los cuales la formación continua es importante según los participantes

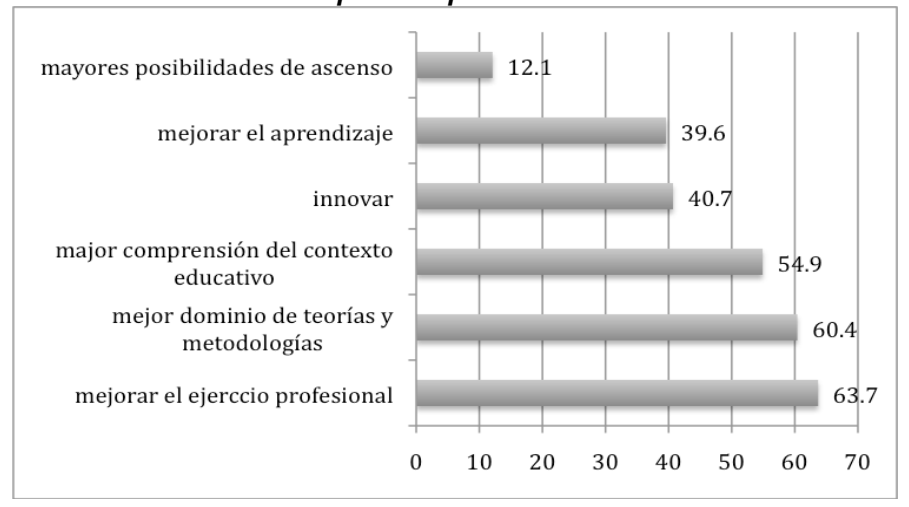

Fuente: Elaboración propia con base en resultados de encuesta a participantes, 2011.

Con el objetivo de conocer los criterios de los graduados alrededor de la importancia de la investigación en su campo laboral, se les pregunta a los entrevistados, si es necesario que los educadores fortalezcan los hábitos de lectura y actualizarse, $72 \%$ dijo estar muy de acuerdo y $26.9 \%$, que de acuerdo. Revista CAES Vol. II, No. 2, Año 2011 ISSN-1659-4703 
Percepción de los graduados de la escuela de ciencias de la educación de la universidad estatal a distancia (UNED) con respecto a la formación recibida durante la carrera

Jensy Campos Céspedes, Jennory Benavides Elizondo

De igual manera se les consulta si estos procesos de enseñanza y aprendizaje en el aula escolar deben enriquecerse con resultados de investigaciones, $70.7 \%$ dice estar muy de acuerdo con lo anterior y el $26.1 \%$ de acuerdo.

Un porcentaje muy similar, $66.7 \%$ está muy de acuerdo en que es importante participar en actividades para elevar la formación en investigación porque esto es un elemento vital para mejorar el ejercicio. Sin embargo, la mayoría de los graduados $39.1 \%$ están de acuerdo que los educadores realizan investigación para desarrollar su práctica docente, pero sin seguir procedimientos científicamente establecidos; un $29.3 \%$ en total acuerdo con lo expresado anteriormente y el $16.3 \%$ ni de acuerdo, ni en desacuerdo.

Los temas que los graduados incluidos en el estudio consideran que deben ser investigados en los contextos educativos costarricenses son los que se consignan en la tabla 3. 
Percepción de los graduados de la escuela de ciencias de la educación de la universidad estatal a distancia (UNED) con respecto a la formación recibida durante la carrera

Jensy Campos Céspedes, Jennory Benavides Elizondo

Tabla 3

Distribución de temas relevantes para investigar de acuerdo con las opiniones de los participantes

\begin{tabular}{lc}
\hline Temas que requieren ser investigados & $\begin{array}{c}\text { Porcentaje } \\
\text { de } \\
\text { opiniones }\end{array}$ \\
\hline Formación pedagógica de los docentes & 53.8 \\
calidad educativa & 51.6 \\
Innovaciones pedagógicas & 51.6 \\
nuevas propuestas pedagógicas para promover pensamiento de orden & 37.6 \\
superior & 58.1 \\
\hline
\end{tabular}

Fuente: Elaboración propia con base en resultados de encuesta a participantes, 2011.

Con respecto al tipo de organización en la que laboran y al tiempo en que tardaron los graduados para conseguir trabajo en el campo profesional del que obtuvieron su título se observó que la mayoría trabaja en el Ministerio de Educación Pública.

Figura 18

Distribución de los participantes de acuerdo con el tipo de organización en la cual laboran

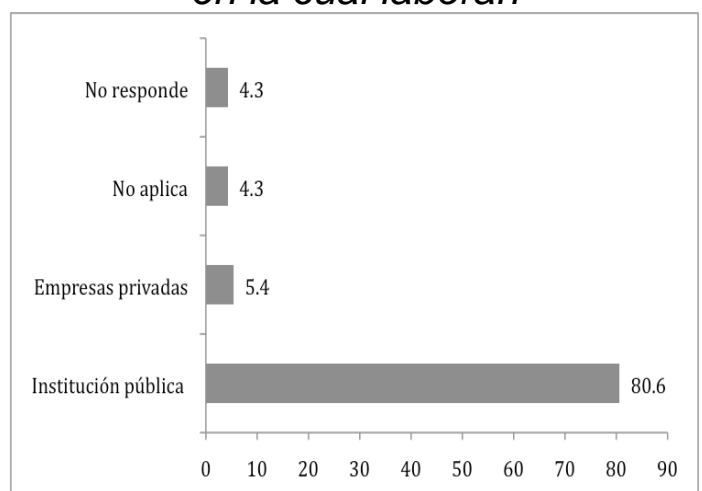

Fuente: Elaboración propia con base en resultados de encuesta a participantes, 2011.
Figura 19

Distribución de los participantes de acuerdo con el tiempo que tardó en conseguir trabajo en el campo en que se graduó

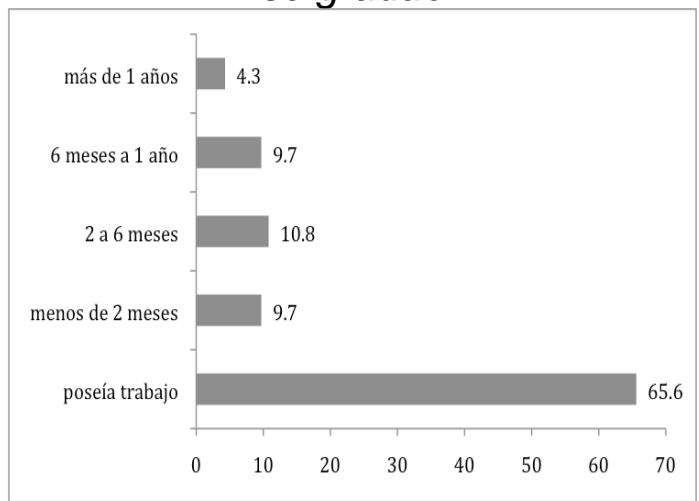

Fuente: Elaboración propia con base en resultados de encuesta a participantes, 2011.

La mayoría de los graduados que participan en el estudio, no debió buscar trabajo porque ya contaban con uno. Lo anterior corrobora que la UNED es una Revista CAES Vol. II, No. 2, Año 2011 ISSN-1659-4703 
Percepción de los graduados de la escuela de ciencias de la educación de la universidad estatal a distancia (UNED) con respecto a la formación recibida durante la carrera

Jensy Campos Céspedes, Jennory Benavides Elizondo

importante opción para quienes están inmersos en el campo laboral, con jornadas completas y en instituciones públicas. Elementos que deben considerarse en el planteamiento de las propuestas académicas.

Con respecto a la opción de continuar estudiando la mayoría de los graduados incluidos en la investigación manifiesta querer continuar con estudios universitarios $76.3 \%$, mientras que $15.1 \%$ indica que no desea estudiar más.

Figura 20

Distribución de los graduados que desea estudiar según grado académico que desea alcanzar

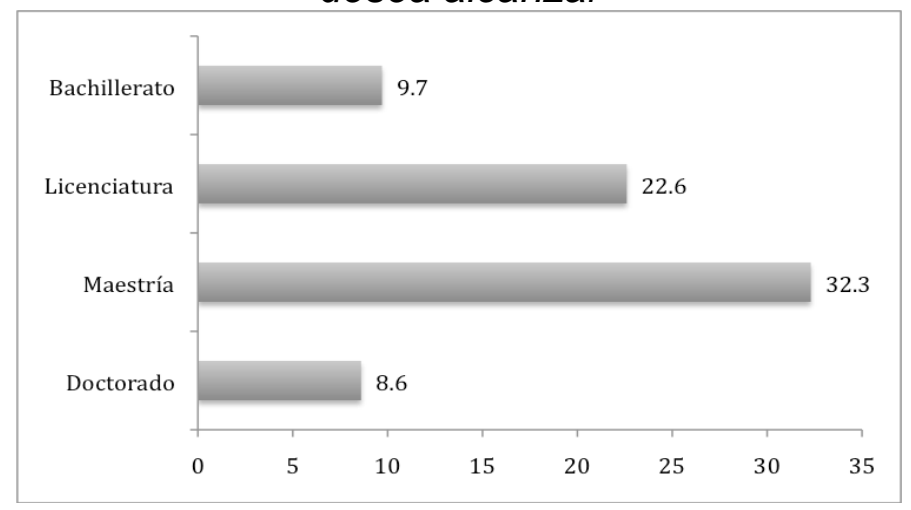

Fuente: Elaboración propia con base en resultados de encuesta a participantes, 2011.

Dado que interesa saber si dentro de estos grados académicos que desean obtener los graduados, están algunos de ellos, relacionados con carreras y especialidades que ofrecen la UNED, tal como se observa en la figura 21. 
Percepción de los graduados de la escuela de ciencias de la educación de la universidad estatal a distancia (UNED) con respecto a la formación recibida durante la carrera

Jensy Campos Céspedes, Jennory Benavides Elizondo

Figura 21

Carreras que los graduados incluidos en el estudio desean estudiar

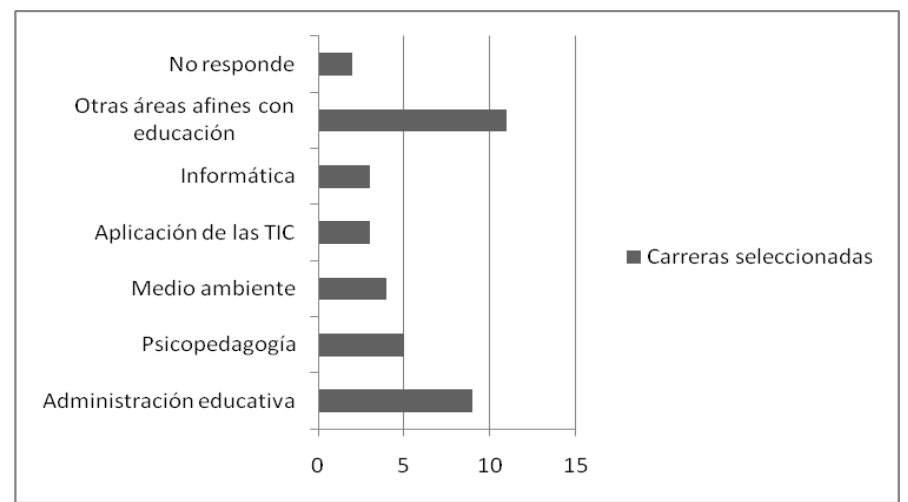

Fuente: Elaboración propia con base en resultados de encuesta a participantes, 2011.

\section{Conclusiones}

Para la ECE resulta importante contar con un flujo de información sistemática sobre el desempeño de los graduados que permita enriquecer y mejorar los planes formativos que se ofertan. Asimismo, es vital establecer vínculos con los centros laborales en los que se ubican los graduados para establecer en ellos centros de práctica para los docentes en formación que cursas las carreras.

Los graduados que han participado en la investigación mostraron interés por participar en actividades académicas y de investigación.

Considerando que los graduados se encuentran dispersos en diferentes zonas del país y que el desarrollo tecnológico ofrece crecientes posibilidades de Revista CAES Vol. II, No. 2, Año 2011 ISSN-1659-4703 
Percepción de los graduados de la escuela de ciencias de la educación de la universidad estatal a distancia (UNED) con respecto a la formación recibida durante la carrera

Jensy Campos Céspedes, Jennory Benavides Elizondo

comunicación la ECE puede planificar actividades dirigidas a los graduados con un uso intensivo de las tecnologías de la información y la comunicación.

Una cantidad importante de los graduados participantes en este estudio mostró interés por el desarrollo de actividades investigativas, ello puede constituirse en un elemento importante para el desarrollo de programas de investigación que permitan conocer a profundidad los escenarios educativos y los resultados de proyectos innovadores que se puedan desarrollar en espacios profesionales donde hoy se insertan los graduados.

Entre las sugerencias, que a partir de este estudio exploratorio se pueden derivar, para estrechar el nexo entre la ECE y los graduados los participantes del estudio indican que se pueden desarrollar las siguientes acciones estratégicas:

- Construir banco de información actualizado de graduados

- Mecanismos divulgación de las actividades de actualización y de formación continua con los graduados

- Construir mecanismos de vinculación universidad -graduado Implementar estrategias que acerquen la universidad al graduado.

- Programas de formación continua.

- Programas de seguimiento a graduados que involucren actividades de profilaxis $u$ autocuidado.

- Fortalecer vínculos y estrategias de coordinación con los empleadores. 
Percepción de los graduados de la escuela de ciencias de la educación de la universidad estatal a distancia (UNED) con respecto a la formación recibida durante la carrera

Jensy Campos Céspedes, Jennory Benavides Elizondo

Para la ECE y en general para la universidad es necesario trabajar en la exploración de diferentes plataformas de aprendizaje en línea que permitan desarrollar oferta de formación continua para los graduados.

Uno de los campos temáticos sobre los que la ECE debe trabajar en la formación continua de sus graduados es el uso pedagógico de los recursos tecnológicos. En especial en el contexto actual cuando la población meta de los graduados de la ECE cada vez tienen mayor acceso y dominio a las tecnologías de la información y la comunicación.

Es imprescindible que por parte de la universidad se tomen decisiones que permitan a las carreras contar con estudios longitudinales de sus graduados mediante los cuales se valore la calidad formativa de la carrera a partir del desempeño profesional de los graduados y no solamente a partir de la percepción de los graduados.

Se requiere contar con programas de seguimiento a graduados que involucren no solo la captura de información respecto algunas variables clave del graduados sino también información de manera sistemática respecto de las necesidades de formación continua, los nuevos escenarios profesionales, los retos y desafíos emergentes para la profesión. 
Percepción de los graduados de la escuela de ciencias de la educación de la universidad estatal a distancia (UNED) con respecto a la formación recibida durante la carrera

Jensy Campos Céspedes, Jennory Benavides Elizondo

\section{Bibliografía}

Argote, L., Duque A., González, L., Payán, A., Payán, C., Rojas, L., et ál. (2001). Los egresados y su desempeño en el medio: un desafío de las instituciones formadoras del recurso humano en salud. Red Colombia Médica. 32: 169173. Recuperado de http://redalyc.uaemex.mx/redalyc/pdf/283/28332403.pdf

Berrocal, V. Brenes, O. Campos, J., Chaves, E., Seas, J. (2008). Informe de autoevaluación Programa Bachillerato y Licenciatura en Informática Educativa. Escuela de Ciencias de la Educación. San José: UNED.

Brenes, O., Campos, J. y Solano, A. (2010). Competencias del docente de educación superior en línea. Revista Actualidades Investigativas. 10 (3) 1 19. Recuperado de http://redalyc.uaemex.mx/redalyc/pdf/447/44717980010.pdf

Campos, J. y Castro, D. (2010). Análisis del estado actual del docente como investigador de su quehacer en Costa Rica: apuntes para la creación de nuevas propuestas de formación docente. Informe de investigación. San José: Universidad Estatal a Distancia- CECC-SICA

Campos, J. y Madriz, L. (2009). Biopedagogía y pertinencia social de la formación docente: nuevas formas de brindar seguimiento a graduados desde los postulados de Paulo Freire. Una experiencia en la UNED de Costa Rica. Ponencia presentada en el Segundo Encuentro Internacional de Investigación Educativa "El pensamiento de Simón Rodríguez y de Paulo Freire".

Campos, J. y Solano, A. (2010). Hacia un modelo de gestión de la oferta educativa en línea. Revista Calidad en la Educación Superior. 1 (2).91-103.

Recuperado de http://www.uned.ac.cr/paa/revista/IIED/HACIA\%20UN\%20MODELO\%20DE \%20Gestión\%20de\%20la\%20Oferta\%20Educativa\%20en\%20Línea.pdf

Carrero Fernández-Baillo. (2008, setiembre 15). Comparar Gestores de Contenidos (CMS). Recuperado de http://carrero.es/comparar-gestores-decontenidos-cms/2188

Fandiño, I. (2008). Una enseñanza e investigación inteligentes de la inteligencia para el éxito escolar y el éxito en la vida cotidiana. Revista Iberoamericana de Educación. 46 (9), 01-12. Recuperado de http://www.rieoei.org/deloslectores/2127Fandinov2.pdf 
Percepción de los graduados de la escuela de ciencias de la educación de la universidad estatal a distancia (UNED) con respecto a la formación recibida durante la carrera

Jensy Campos Céspedes, Jennory Benavides Elizondo

UNESCO. (2005). Hacia las sociedades del conocimiento. Informe Mundial. París: Ediciones UNESCO. Recuperado de http://unesdoc.unesco.org/images/0014/001419/141908s.pdf

Sánchez, E. (2010). Resultados generales de la encuesta aplicada a las personas graduadas durante el 2008. Centro de Investigación y Evaluación Institucional. UNED. 